\title{
An individually tailored family-centered intervention for pediatric obesity in primary care: study protocol of a randomized type II hybrid effectiveness-implementation trial (Raising Healthy Children study)
}

\author{
Justin D. Smith ${ }^{1,2,3^{*}}$ (D, Cady Berkel ${ }^{4}$, Neil Jordan ${ }^{1}$, David C. Atkins ${ }^{5}$, Shrikanth S. Narayanan ${ }^{6}$, Carlos Gallo ${ }^{1}$,
} Kevin J. Grimm ${ }^{4}$, Thomas J. Dishion ${ }^{4}$, Anne M. Mauricio ${ }^{4}$, Jenna Rudo-Stern ${ }^{4}$, Mariah K. Meachum", Emily Winslow ${ }^{4}$ and Meg M. Bruening ${ }^{7}$

\begin{abstract}
Background: Pediatric obesity is a multi-faceted public health concern that can lead to cardiovascular diseases, cancers, and early mortality. Small changes in diet, physical activity, or BMI can significantly reduce the possibility of developing cardiometabolic risk factors. Family-based behavioral interventions are an underutilized, evidence-based approach that have been found to significantly prevent excess weight gain and obesity in children and adolescents. Poor program availability, low participation rates, and non-adherence are noted barriers to positive outcomes. Effective interventions for pediatric obesity in primary care are hampered by low family functioning, motivation, and adherence to recommendations.

Methods: This (type II) hybrid effectiveness-implementation randomized trial tests the Family Check-Up 4 Health (FCU4Health) program, which was designed to target health behavior change in children by improving family management practices and parenting skills, with the goal of preventing obesity and excess weight gain. The FCU4Health is assessment driven to tailor services and increase parent motivation. A sample of 350 families with children aged 6 to 12 years who are identified as overweight or obese (BMI $\geq 85$ th percentile for age and gender) will be enrolled at three primary care clinics [two Federally Qualified Healthcare Centers (FQHCs) and a children's hospital]. All clinics serve predominantly Medicaid patients and a large ethnic minority population, including Latinos, African Americans, and American Indians who face disparities in obesity, cardiometabolic risk, and access to care. The FCU4Health will be coordinated with usual care, using two different delivery strategies: an embedded approach for the two FQHCs and a referral model for the hospital-based clinic. To assess program effectiveness (BMl, body composition, child health behaviors, parenting, and utilization of support services) and implementation outcomes (such outcomes as acceptability, adoption, feasibility, appropriateness, fidelity, and cost), we use a multi-method and multi-informant assessment strategy including electronic health record data, behavioral observation, questionnaires, interviews, and cost capture methods.

(Continued on next page)
\end{abstract}

\footnotetext{
* Correspondence: jd.smith@northwestern.edu

Justin D. Smith and Cady Berkel contributed equally to the production of this manuscript and to the conception of the study protocol. Justin D. Smith and Cady Berkel are co-principal investigators of the study.

'Department of Psychiatry and Behavioral Sciences, Northwestern University

Feinberg School of Medicine, Chicago, IL, USA

${ }^{2}$ Department of Preventive Medicine, Northwestern University Feinberg

School of Medicine, Chicago, IL, USA

Full list of author information is available at the end of the article
} 
(Continued from previous page)

Discussion: This study has the potential to prevent excess weight gain, obesity, and health disparities in children by establishing the effectiveness of the FCU4Health and collecting information critical for healthcare decision makers to support sustainable implementation of family-based programs in primary care.

Trial registration: NCT03013309 ClinicalTrials.gov

Keywords: Family Check-Up 4 Health, Primary care, Hybrid effectiveness-implementation trial, Pediatric obesity, Integrated care, Coordinated care

\section{Background}

Childhood obesity has become the leading preventable cause of death worldwide [1]. Poor diet/nutrition and physical inactivity are the leading and preventable contributors to obesity and are among the principal causes of chronic disease and mortality for youths [2]. Obesity is also related to psychosocial issues in youth, such as depression and academic disengagement [3-5]. In the USA in 2013-2014, it was estimated that $18 \%$ of all 6 -11-year-old children were obese and that a disproportionate number are disadvantaged economically and socially [6]. Obesity and its health consequences are disproportionately distributed across the USA; Mexican Americans, American Indians, and African Americans have the highest prevalence rates of obesity in childhood at 41.1 to $56.3 \%$ [6-9]. Social determinants of health [10] play a key role in the childhood obesity and contribute substantially to disparities. In the social environment, stress and discrimination are acutely experienced by minority and low-income communities in the USA and are linked with childhood obesity [11]. Physical environment barriers include limited access to safe areas for play and fresh, nutritious foods [12]. Finally, access to health services that can prevent obesity and its sequelae [13] is limited for members of underserved groups [14].

While addressing the social determinants of health is an ongoing task, significant evidence supports the effectiveness of lifestyle modification on cardiometabolic risk factors, with even small changes yielding significant impact [15]. Recently published reviews $[16,17]$ indicate that youth health behaviors and obesity may be improved when parents attend and are directly involved with services [18] and are provided training in the skills required to support lifestyle modification in accordance with expert guidelines $[16,19-21]$.

Primary care is a promising context for improving parenting behaviors linked to child health [22-24]. Most children access a primary care provider annually $[25,26]$, caregivers are present at visits, and physicians have a high level of perceived authority among parents who look to them for advice on children's health. The National Academy of Medicine, the American Academy of Pediatrics, and the Endocrine Society, among others, promote a family-centered intervention in primary care [27-29]. Despite the appeal of a familycentered approach [30, 31], several barriers hinder implementation of such programs in real-world healthcare systems [29, 32]. For example, healthcare providers report inadequate time, training, and resources to effectively work with parents to address childhood obesity [33]. Few comprehensive and prospective evaluations of the implementation of weight management programs in primary care have been done, despite such factors as inadequate cost evaluation being a top reason for failure to adopt behavioral interventions [34] and the general concern about delivery with sufficient fidelity to preserve effectiveness [35]. To have a population impact on pediatric obesity, there is a pressing need for scalable programs that can be delivered in primary care and engage parents in behavior change strategies for children [32].

\section{The Family Check-Up for Health program}

The Family Check-Up 4 Health (FCU4Health) is an adaptation and enhancement of the original Family Check-Up (FCU), an evidence-based, family-centered intervention with empirical evidence of effectiveness and feasible delivery in real-world systems that provide care for culturally diverse, low-income families [36-39]. Initially designed and tested as a behavioral health intervention, the FCU employs motivational interviewing (MI) to engage parents in family management strategies and community-based support services [40]. Extensive data document the FCU's clinical effectiveness and high rates of engagement among underserved and ethnic minority families [36-38, 41-43]. Although not targeted by the program, the FCU demonstrated long-term collateral effects on nutrition (ages 2-5) and obesity (ages 2 to 10 years) [44] and on those from adolescence to early adulthood (ages 12 to 22 years) [13, 44]. Improvement in parent-child relationships and positive behavior support mediated the effects of the FCU on nutrition and obesity in both developmental periods. Based on these findings, the FCU4Health was developed to specifically target pediatric obesity in primary care, the front-line service setting for prevention of obesity and excess weight gain [29]. This involved enhancing the content to focus on helping parents support child and family behaviors that lead to a healthy weight, and adapting the delivery strategy for the primary care context (Smith JD, Berkel C, Rudo-Stern J, Montaño Z, Mauricio AM, Dishion TJ, St. George SM, Prado G, Chiapa A, Bruening MM, The Family Check-Up 4 Health (FCU4Health): The process of adapting an 
evidence-based parenting program for prevention of pediatric obesity and excess weight gain in primary care, submitted).

The FCU4Health is unique relative to other obesity interventions in that rather than solely targeting knowledge about national health guidelines (published by such organizations as the American Academy of Pediatrics and United States Department of Agriculture that require updating over time), it provides parents with the skills and support to implement the changes recommended by physicians, dieticians, and other members of the healthcare team. This aspect fills an important gap for families who earnestly agree to improve children's diet, physical activity, sleep habits, or screen time while in the clinic but encounter difficulties in their regular involvement that lead to an abandonment of these efforts. It also increases the individualizability of the intervention to the needs of the family and reduces training time and the need for regular updates to program materials.

The core components of the FCU4Health mirror those of the original FCU: an ecological assessment of the family, a feedback and motivation session, and follow-up services that are individually tailored in content and dosage based on identified needs in the assessment (see Fig. 1). These follow-up services may include parenting modules for needs related to family management and/or care coordination with community-based services to address ecological needs [38]. Parenting modules follow Everyday Parenting, [45] a 12-module skills-based curriculum focusing on the three core areas of parenting and family management: relationship quality, positive behavior support, and monitoring and limit setting. The application of these steps in this trial is described in greater detail in the "Methods" section.

\section{Study design}

\section{Overview}

We will test the FCU4Healtth with a type II hybrid effectiveness-implementation trial [46], which enables simultaneous evaluation of the clinical effectiveness and the delivery of the FCU4Health program using two different implementation strategies [47].

\section{Implementation strategies and service delivery sites}

The delivery model used by a given practice depends upon their programmatic, fiscal, and resource characteristics and needs [48]. Several approaches for the delivery of behavioral health services in primary care settings have been described [49]. In this trial, we partnered with three pediatric primary care agencies that serve high rates of lowincome, ethnic minority families (about 60-65\% Latino; 10\% African American; 5-8\% American Indian). Because pediatric primary care offices often do not have integrated behavioral health, arm 1 uses a strategy of coordinated care between primary care and an external behavioral health provider. We partnered with a large outpatient general pediatrics clinic associated with a children's hospital. We also partnered with two agencies that are part of the nationwide network of 1250 Federally Qualified Health Centers (FQHCs). A feature of FQHCs is the integrated care model that typifies the medical home. In arm 2, we will test an integrated/co-located model of care. The FQHC network is crucial for embedding a family-centered approach to care because it serves youth and families at high sociodemographic risk and is intended to integrate services to reduce barriers and improve care. Partnering with these provider sites afforded the opportunity to compare FCU4Health implementation under two different real-life service delivery models. In both arms, behavioral health consultants are responsible for delivering the FCU4Health and coordinating care with other support services and resources.

\section{Clinical effectiveness of the FCU4Health}

Within the two implementation strategy arms, eligible families are randomized to receive either the FCU4Health or clinic services-as-usual plus community program information (see "Methods"). Primary clinical outcomes are child BMI and body composition. Secondary outcomes, hypothesized to mediate effects of the intervention on primary outcomes, are lifestyle behaviors (diet and nutrition, physical

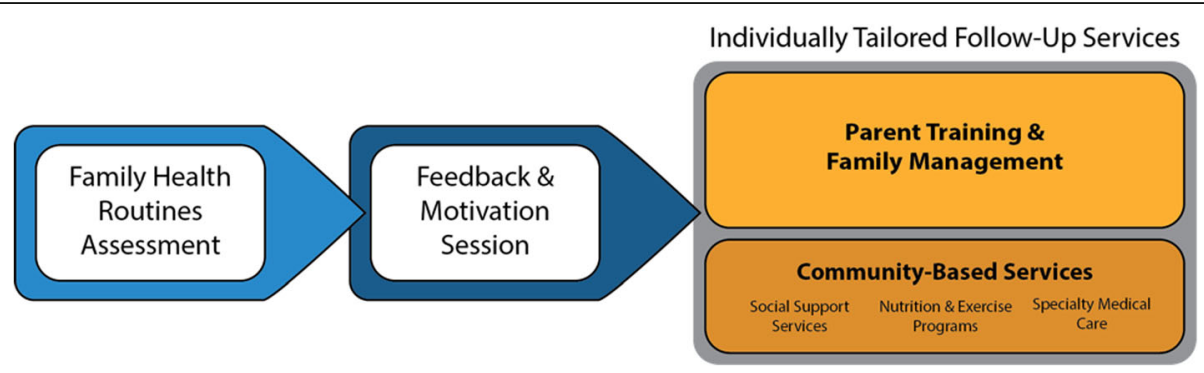

Fig. 1 Steps and elements of the Family Check-Up for Health (FCU4Health) program in the Raising Healthy Children study 
and sedentary activity, sleep), child self-regulation, and family management practices.

\section{Methods}

\section{Study aims}

Aim 1

Aim 1 was to finalize the adaptation of the FCU4Health, which was initially adapted and piloted in pediatric primary healthcare [50], based on input from a community advisory board $(\mathrm{CAB})$ and partner clinics.

\section{Aim 2}

Aims 2 was to evaluate the effects of two delivery strategies: integrated/co-located care and coordinated care with referral. Using a mixed-methods approach applied to Proctor et al.'s $[51,52]$ taxonomy, we will evaluate the process of implementing the FCU4Health to inform, taking the program to scale.

2a. Evaluate fidelity over time to the FCU4Health using a validated observational rating system [53-55] and develop and test an automated fidelity coding system [56].

2b. Employ behavioral intervention costing methods

[57] to evaluate the costs of installing and delivering the FCU4Health and conduct a cost-benefit analysis to evaluate the monetary benefits of program effects.

2c. Develop a plan to support scale-up and sustainment in collaboration with our CAB.

\section{Aim 3}

Aim 3 was to use pragmatic trial principles to test program effects on primary and secondary outcomes. Intervention effects on proximal outcomes will be tested as mediators on the distal outcomes of child BMI and body composition.

\section{$\operatorname{Aim} 4$}

Aim 4 was to carefully track utilization in each aspect of the program, gather data concerning family's service delivery preferences and satisfaction, and test a model of program implementation where family engagement mediates the relation between fidelity and clinical outcomes [58].

\section{Study participants, recruitment, and randomization}

Families with children 5.5 to 12 years of age with BMI $\geq$ 85th percentile for age and gender at study entry are eligible to participate. Participants will be identified during clinic well- and sick-child visits and through queries of the Electronic Health Record (EHR). Enrolled families will complete an ecological, family health routine assessment. After the first assessment, families will be randomly assigned to the FCU4Health $(n=200)$ or Services-asUsual Plus Information condition $(n=150)$ using a stratified block randomized design by child gender, age, language (English, Spanish), and ethnicity. Assessing the family prior to randomization ensures double-blinding at baseline. Families in the intervention condition will participate in the FCU4Health program, as outlined in the next section, in addition to receiving usual care through their clinic. Families are compensated for completing assessments, but not for engaging in services. A CONSORT table is presented in Fig. 2.

\section{Family Check-Up 4 Health program Delivery schedule}

Previously, the FCU has used a health maintenance approach, in which parents receive periodic (annual) FCUs $[38,59]$. Based on the US Preventive Services Task Force recommendations that overweight/obese youth should be provided with at least moderate treatment dosage to affect weight management [60], we designed a delivery schedule to achieve a minimum targeted dose of intervention between 25 and 50 h over 6 months (assessment and feedback at months 1, 3, and 6). Because of the tailored approach, families with greater need and those with greater motivation are expected to participate in more follow-up services [61].

\section{FCU4Health family health routine assessment}

The family health routine assessment is ecological and evaluates five broad domains-family health behaviors, child health behaviors, family well-being and support, child adjustment, and family management and relationships-using a multi-agent battery of caregiver- and child-reported questionnaires and a series of brief videorecorded family interaction tasks (FITs). Two versions of the assessment are used in this study: a full version at months 1,6 , and 12 (90 min to complete) and a brief version administered only at the 3-month assessment (30 min). The full version includes all questionnaires across the five broad domains and three FITs on the topics of health and diet goals, monitoring and limit setting of child health behaviors, and planning a fun physically active family activity ( $4 \mathrm{~min}$ each). The brief version includes only the questionnaires to caregiver and child pertaining to family health behaviors and child health behaviors and is intended as a low-burden opportunity to reinforce steps toward behavior change and address areas that have not yet improved or potentially shift focus to areas that have emerged during the first 3 months of intervention. Questionnaires are completed in English or in Spanish by caregivers and the child through a secure website accessed via a Wi-Fi enabled tablet. Pencil-and-paper versions are available if needed. FITs are recorded using the tablet and are uploaded to a cloud-based, HIPAA-compliant portal for secure storage, viewing, and scoring. 


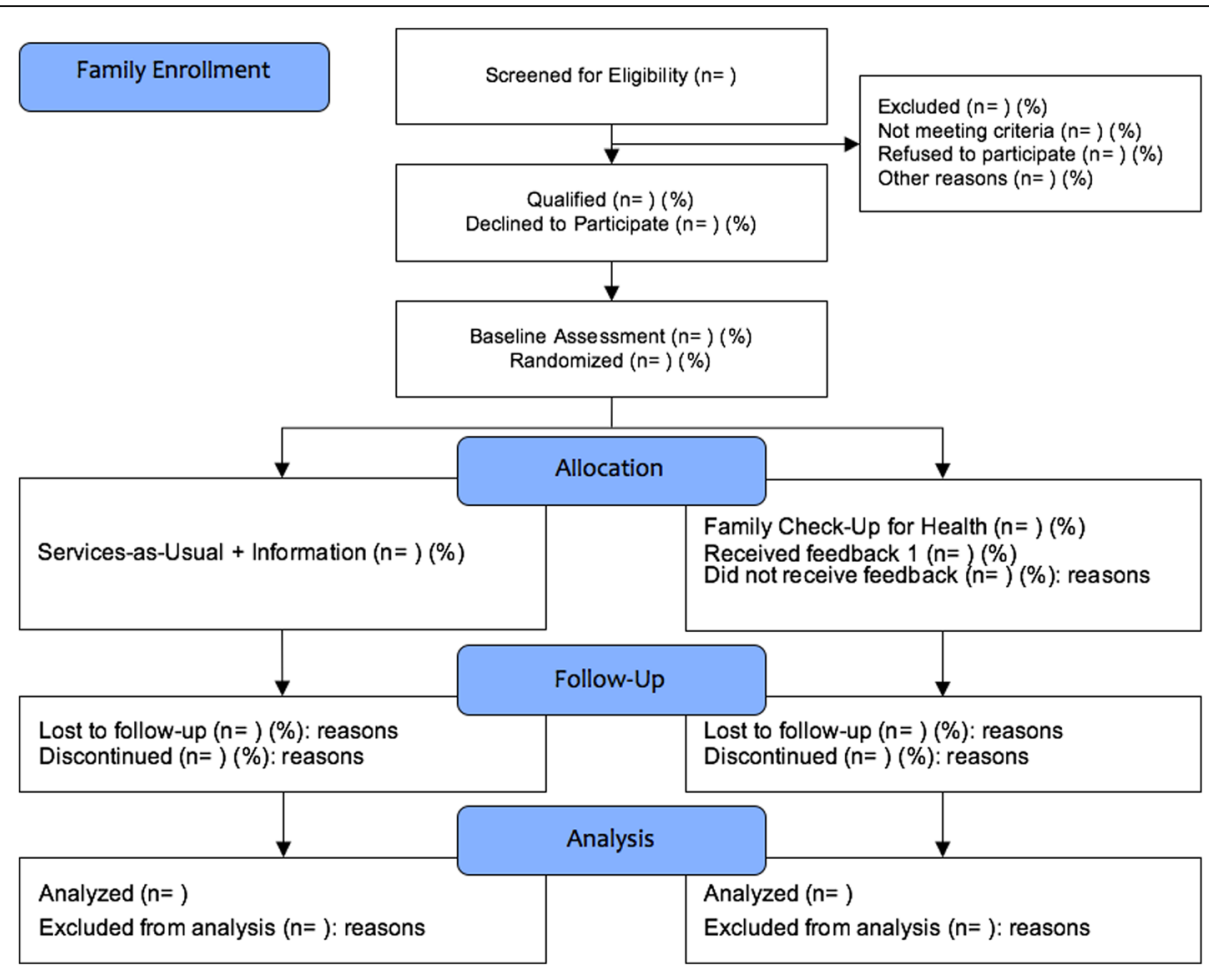

Fig. 2 CONSORT flow diagram

\section{Feedback sessions}

Feedback sessions follow the assessments in months 1, 3 , and 6 . The first feedback session begins with a discussion to understand (a) the caregivers' perception of their needs; (b) their child's health, adjustment, and obesogenic behaviors; and (c) the caregivers' motivation to change parenting and family management practices in support of health behavior change. In the second and third feedbacks, the coordinator begins by checking in with the family about their progress, discussing barriers they are experiencing, and exploring the ways that the previous feedback and Everyday Parenting sessions were helpful for them in catalyzing and supporting healthy lifestyle behavior change. The coordinator presents the findings of the family health routine assessment using the Feedback Form, which summarizes where families are in the five areas of the assessment relative to agebased norms with a stoplight color scheme. Family strengths are represented in green, yellow indicates areas to monitor, and red accentuates areas in need of further support. The goals of this session are to (a) share assessment findings with family members regarding strengths and challenges, (b) engage in a motivation-enhancing discussion about promoting positive changes, and (c) provide a menu of resources and next steps for intervention. The first two feedback sessions lay out a plan for the follow-up services (i.e., Everyday Parenting sessions or referrals to community-based services) and use MI techniques to increase buy-in for the planned course of action. In the final feedback session (month 6), feedback and motivation focus on the progress the family has made and continuing engagement in community services to address areas in need of additional support.

\section{Everyday Parenting sessions}

In the FCU4Health, Everyday Parenting sessions are tailored to the family's specific needs identified in the family health routine assessment and focus on a specific behavior change goal, such as setting limits on snacking between family meals or monitoring children's sedentary and physical activity time. It is expected that families will engage in 8-16 sessions of Everyday Parenting over the 6-month intervention period. The focus is on supporting and maintaining children's healthy behaviors. It is also expected that during these sessions, the FCU4Health coordinator will help the caregivers problem-solve challenges they have encountered in implementing recommended health behavior changes.

\section{Community programs and support services}

To address needs related to health behaviors (e.g., nutrition and physical activity programs), specialty medical needs (e.g., asthma, diabetes), and the social determinants of health that affect parents' ability to support child health (e.g., employment, food and housing insecurity, insurance coverage), coordinators provide families with referrals to 
existing resources in the community. We will identify programs in collaboration with our partner agencies that are available at little or no cost to families. The intent is to leverage existing services to support families and to test whether the motivational aspects of the FCU4Health lead to increased engagement compared to families in usual care. A centralized repository of available programs and their requirements is stored in a Web-based hub, which can be updated by coordinators and other staff.

\section{Phone-based coaching}

In weeks where a face-to-face session is not scheduled or does not occur (e.g., cancelation, no show), the coordinator or a behavioral health support staff person (e.g., a community health worker) conducts a 15 - to 30-min phone-based coaching session. The purpose is to maintain contact with the family and help problem-solve challenges, reinforce positive achievements, and continually address motivation to change and barriers to engagement. Additional information about the program is available in the FCU4Health protocol manual [62].

\section{Services-as-Usual Plus Information condition}

Families randomized to the services-as-usual arm will receive care as usual from their primary care provider and any additional services offered by the agency, as well as a listing of the same community resources offered to families randomized to the FCU4Health arm.

\section{Measures}

Additional information on measures of implementation and clinical effectiveness is presented in Tables 1 and 2, respectively.

\section{Implementation}

To assess the acceptability, feasibility, appropriateness, and sustainability of each component of the FCU4Health (e.g., training, program costs, content, delivery), an agency stakeholder battery will be electronically administered to at least 30 agency stakeholders (approximately ten from each agency) at two times during the study: 4 months into the FCU4Health delivery and when all families enrolled in the study complete the entire 6month FCU4Health protocol. Families in the FCU4Health arm will rate the acceptability and appropriateness of the program via an electronically administered survey at months 6 and 12. Additionally, 20\% of families in the FCU4Health arm will be randomly selected and interviewed by study staff via phone.

Study enrollment and participation data will be used to evaluate the reach of the program in each agency by tracking both the proportion of eligible families referred to the study and the number randomized to the FCU4Health condition that engage in the program compared with the total number of eligible families [63].

We will assess the fidelity of coordinators to the FCU4Health using an established observation rating system that defines high-quality care and was associated with clinical improvement in studies of the original FCU [53, 54]. A trained coding team will code video or audio recordings of the first FCU4Health feedback session from every family $(N=200)$, and a subset of 80 families will be randomly selected at study entry, balanced by coordinator, for longitudinal coding of all three FCU4Health feedback sessions to assess drift ( $N=160$ additional sessions). In addition, we will develop an automated coding system based on an existing, validated automated coding system for motivational interviewing. Automated fidelity coding will be built from previous methodologies developed for coding fidelity to MI (presence of complex reflections, open-ended questions) $[56,64,65]$ and family interventions [66, 67]. Machinegenerated fidelity codes have been found to be reliable with human coding across multiple studies [56, 64-66, 68, 69]. Initial algorithm training will use existing FCU feedback session recordings and the FCU fidelity codebook. Automated coding involves a computational "pipeline" of both speech signal processing and machine learning of the audio recordings of the conversations during the sessions, including (1) voice activity detection, (2) diarization (i.e., how many speakers are in session and when is each talking?), (3) role identification (i.e., who is the provider?), (4) automatic speech-to-text transcription, and (5) machine learning models to predict fidelity codes, using lexical (i.e., words) and paralinguistic features as inputs.

Cost data collection is multi-method in order to perform analyses from multiple perspectives (e.g., agency, payor, family). Study staff report activities weekly on an electronically administered survey. FCU4Health coordinators complete a brief survey after each contact with study families (in the intervention arm) to capture the staff involved and the number of hours spent. Electronic budgets track program spending and will be used to prospectively separate costs associated with implementation from those specifically related to research (e.g., participant reimbursement, data analysis software), start-up (e.g., training), and ongoing costs (e.g., consultation with FCU4Health developers, technical assistance, travel for home visitation). At each assessment, families will be asked to provide a release for the study team to obtain health plan claims data, which will be used to identify relevant health services utilization and associated costs.

Critical to the study's implementation aims, such as the calculation of reach and cost, is the careful tracking of participation in the various components of the program. Using methods developed and tested in previous trials, the FCU4Health coordinators and other agency staff will track 
Table 1 Implementation outcomes

\begin{tabular}{|c|c|}
\hline Outcome variable(s) & Measure(s) and data collection procedures \\
\hline \multirow{2}{*}{$\begin{array}{l}\text { Stakeholders: acceptability, feasibility, } \\
\text { appropriateness, and sustainability }\end{array}$} & (1) Select scales of the Annual Survey of Evidence-Based Programs [113] $(a>.75)$ \\
\hline & $\begin{array}{l}\text { (2) FCU4Health Stakeholder Survey: } 11 \text { open-ended questions, adapted from the } \\
\text { Treatment Acceptability Rating Form, related to the relevance of the FCU4Health } \\
\text { for obesity management, barriers and facilitators of the delivery of the program, } \\
\text { and feasibility of this program from the perspective of stakeholders }\end{array}$ \\
\hline
\end{tabular}

(3) Program Sustainability Assessment Tool [114]: 8 domains (e.g., funding stability, organizational capacity) with 5 items each (e.g., "The program has sustained funding") are rated on a 7-point Likert scale ( $1=$ little or no extent, $7=$ a very great extent) $(a=.88)$

Caregivers: acceptability and appropriateness

Reach

Family service participation

Fidelity

Costs and health economics

(1) FCU4Health activities checklist (FACL) [118]
(1) FCU4Health Caregiver Acceptability Interview, designed for and used in our pilot feasibility trial [50], consists of 11 open-ended questions pertaining to the relevance of the program components to the family's efforts to manage weight, the acceptability of the program and its components, and the barriers and facilitators of participation

(2) Treatment Acceptability Rating Form-Revised Short [115-117], adapted for FCU4Health: 10 items (e.g., "How likely is FCU4Health to make permanent im provements in your child's health behaviors?") rated on a 7-point Likert scale (1 = not at all, $7=$ very) $(a=.92)$ [115]

1- or 2-month sampling period to more closely approximate the number of families that require a service at any given point [63] (see description in the "Methods" section)

(2) Community Resources: Engagement and Adequacy (CREA): adapted from an existing care coordination measure to assess the extent to which families engaged in community resources (e.g., emergency care, well-child visits, recreational and nutrition programs, mental healthcare, school services, financial services) and if help was needed to obtain the resource and whether those resources met their needs. Response options are as follows: "I didn't need help"; "I needed help, but didn't find it"; "I tried this, but it didn't work"; "I'm still getting help"; and "I got help, and it worked"

(3) Dosage of Engagement in Community Resources (DECR) [119]: created for this study to assess the amount of time spent in activities to support health behaviors assessed by asking the number of times among 7 response options (e.g., "once a month," "2-3 times a week," "2 times every day") and then the duration of each instance of teach activity using a drop-down menu of min (e.g., 30) to $\mathrm{h}$ (e.g., 1, 3, and $8 \mathrm{~h}$ or more)

(1) COACH observational rating system [54]: 5 dimensions of observable in-session coordinator skills: conceptual accuracy; observant and responsive to the families' contexts and needs; actively structures session to optimize effectiveness; carefully teaches and provides corrective feedback; hope and motivation are generated. Each dimension contains exemplars (prescribed behaviors) and non-exemplars (proscribed behaviors) and is rated on a 9-point scale: 1-3 (needs work); 4-6 (competent work); 7-9 (excellent work) (ICC $\geq$.73) [120]. Variability in fidelity ratings to feedback sessions have been associated with long-term changes in parenting skills and child behavioral outcomes [53-55]

(2) Automated coding of fidelity is being developed within this study (aim 2b)

(1) Cost capture survey [57]

Automated coding

Survey (ST)

Survey (CO)

FCU4Health activities checklist (FACL) [118]

(see description in the "Methods" section)

(3) Electronic budgets

Administrative data

Administrative data

CG caregiver, CO FCU4Health coordinator, ST stakeholder, EHR electronic health record

all family contacts and record the delivery location, travel time, details of participating family members, the type of the FCU4Health session, content areas covered, and referrals. At each assessment, caregivers will report on their engagement in community resources, how well these resources met their needs, and the amount of time spent in activities to support health child and family behaviors. 
Table 2 Clinical effectiveness and related child and family outcomes

\begin{tabular}{lll}
\hline Outcome variable(s) & Measure(s) and data collection procedures & $\begin{array}{l}\text { Data source and reporter } \\
\text { (when applicable) }\end{array}$ \\
\hline $\begin{array}{l}\text { Child weight and } \\
\text { body composition }\end{array}$ & $\begin{array}{l}\text { Portable electronic scale (Tanita SC-331SU) approved by the FDA for BMI and body } \\
\text { composition in children (ages } 5 \text { to 18) and adults. Child BMI is standardized by sex } \\
\text { and age according to the CDC growth reference data for children [121] }\end{array}$ & Anthropometric \\
Child dietary habits & $\begin{array}{l}\text { NHANES Dietary Screener Questionnaire [122]: 9-point scale }(0=\text { never, } 8=6 \text { or more times } \\
\text { per day) to rate } 3 \text { items about fruit, vegetable, and fast-food choices (e.g., "In the past month, }\end{array}$ & Survey (CG)
\end{tabular}
per day) to rate 3 items about fruit, vegetable, and fast-food choices (e.g., "In the past month, how often did you eat fruit?") and 3 items regarding sugar-sweetened beverage choices (e.g., "In the past month, how often did you drink regular soda that contained sugar?")

Family health routines and (1) Family Health Behaviors Scale [123]: 24 items rated on a 5-point scale ( $0=$ almost never, health behaviors

$4=$ nearly always; e.g., "I participate in physical activity with [child name]; [child name] sneaks food"). Caregiver ratings on this scale are sensitive to change and have been shown to predict child weight classification

(2) Sleep parenting routines: 6 items on a 5-point scale $(0=$ almost never, $4=$ nearly always; e.g.. [child name] goes to bed at about the same time each night). Items developed from existing measures, such as the Brief Infant Sleep Questionnaire [124]

(3) Media parenting routines: 6 items on a 5-point scale $(0=$ almost never, $4=$ nearly always; e.g., "I keep track of [child name]'s screen-time") and a single question on $\mathrm{h}$ per day of media use. Items were drawn from published studies and measures [125]

Parenting and family management skills
Child self-regulation

Body image

Quality of life
Questionnaires encompass 3 domains of parenting and family management skills:

(1) positive behavior support, (2) relationship quality, and (3) monitoring and limit setting. Each of these measures has been used in previous FCU trials and was found to have adequate reliability, internal consistency, and sensitivity to change

Caregiver: 5 -point scale $(0=$ never, $4=$ very often) on the domains of incentives and encouragement (4 items; e.g., "Gave [child name] a hug, kiss, or kind word") [126]; proactive parenting (7 items; e.g., "Plan for ways to prevent problem behavior") [127], parent-child conflict (10 items; e.g., "[child name] gets angry at me easily"); family conflict (5 items; e.g.,

"We got angry at each other") [126]; quality time (6 items; e.g., "Involve [child name] in household chores") [127], parent warmth (5 items; e.g., "If upset, [child name] seeks comfort from me") [128]; family routines (7 items; e.g., "Check to see if [child name] has homework"); limit setting (7 items, e.g., "Speak calmly with [child name] when you were upset with him/her"), negative parent behavior (5 items; e.g., "Criticize [child name]") [127]; and a single question on $\mathrm{h}$ per day of unsupervised time

Child: 4 items on incentives and encouragement (e.g., "Praised you or complimented you for something you did well"), using a 5 -point scale $(0=$ never, $4=$ very often); 4 -item questionnaire on family conflict (e.g., "I got my way by getting angry"), using a 7-point scale $(0=$ never, $6=8+$ times $)[126]$

Family interaction task (FIT) observational coding system [129]: the recorded family interactions are scored for caregiver(s) behaviors in the domains of relationship quality, positive behavior support, and monitoring and limit setting, as well as demonstrated knowledge of children's health behaviors (e.g., age-appropriate physical activity duration and dietary guidelines). Child behaviors and emotional adaptation are rated. Each domain is rated for parent's skill/knowledge on a 5-point scale ( $1=$ low, $5=$ high) for each interaction task independently

Caregiver: 13-item survey (e.g., "[child name] is able to resist laughing or smiling when it isn't appropriate") adapted from the Children's Behavior Questionnaire [130], using a 5-point scale ( $1=$ almost always untrue, $5=$ almost always true)

Child: 16-item questionnaire (e.g., "I pay close attention when someone tells me how to do something") adapted from the Early Adolescent Temperament Questionnaire [131], using a 6-point scale ( $1=$ always untrue, $5=$ always true $)$

Perception of Teasing Scale [132]: children will use a 5-point scale ( $1=$ never/not upset, $4=$ very often/extremely upset) to rate the frequency of 3 events pertaining to weight-related stigma and describe their level of distress associated with these items (e.g., "People made fun of you because you were heavy"; "How upset does this make you?")

Body Image Scale for Children: a pictorial scale using body pictures representing standardized percentile curves of BMI for boys and girls, separately; good reliability and evidence of validity with children aged 7 to 12 [133]; proxy for satisfaction and a measure of potential adverse effects of participation in the program/study

Pediatric Quality of Life Inventory [134]: 23 items in four categories: physical functioning, emotional functioning, social functioning, and school functioning

Caregivers: 5 -point scale $(0=$ never, $4=$ almost always) to rate items (e.g., "[child name] feels afraid or scared"; "[child name] gets along with other children")

Children: 3 -point scale $(0=$ not at all, $2=$ somewhat, $4=a$ lot) to rate items (e.g., "It is hard for me to run"; "It is hard for me to pay attention in school") adjusted for 2 age groups: $5-7$ years old and $8-12$ years old
Observational

Survey $(\mathrm{CG}, \mathrm{CH})$
Survey $(\mathrm{CH})$ 
Table 2 Clinical effectiveness and related child and family outcomes (Continued)

\begin{tabular}{|c|c|c|}
\hline Outcome variable(s) & Measure(s) and data collection procedures & $\begin{array}{l}\text { Data source and reporter } \\
\text { (when applicable) }\end{array}$ \\
\hline \multirow[t]{2}{*}{ Satisfaction with care } & $\begin{array}{l}\text { (1) Family Check-Up Caregiver Service Satisfaction Survey: } 9 \text { items rated on a 4-point scale } \\
(0=\text { strongly disagree, } 4=\text { strongly agree) adapted from the Client Satisfaction Questionnaire } \\
\text { [135] to be specific to parent training programs. This was developed for use with the original } \\
\text { FCU conducted in community mental health clinics [89] }(a=.95) \text { in that trial }\end{array}$ & Survey (CG) \\
\hline & $\begin{array}{l}\text { (2) Parent Experience of Assessment Survey (PEAS) [136]: } 3 \text { of the } 5 \text { subscales } \\
\text { (parent-coordinator collaboration, systemic awareness, and negative feelings) } \\
\text { with } 15 \text { total items rated on a } 5 \text {-point scale } \\
\text { (1 = strongly disagree, } 5=\text { strongly agree) }(a>.75)\end{array}$ & \\
\hline Child adjustment & $\begin{array}{l}\text { Strengths and Difficulties Questionnaire [137]: caregivers and children rate } 5 \text { items each on a } \\
\text { 3-point scale }(0=\text { not true, } 1=\text { somewhat true, } 4=\text { very true) on the conduct problems } \\
\text { (e.g., "I/[child name] often lose(s) temper"), hyperactivity (e.g., "I am/[child name] is constantly } \\
\text { fidgeting or squirming"), pro-social behavior (e.g., "I am/[child name] is considerate of other } \\
\text { people's feelings"), and emotional problems (e.g., "I am/[child name] is often unhappy, } \\
\text { depressed, or sad") subscales }\end{array}$ & Survey $(\mathrm{CG}, \mathrm{CH})$ \\
\hline
\end{tabular}

$\mathrm{CH}$ child, CG caregiver, CO FCU4Health coordinator, ST stakeholder, EHR electronic health record

\section{Effectiveness}

At each assessment, the child and caregiver(s) will be weighed using a portable electronic scale. $B M I$ is the primary study outcome because it indicates cardiometabolic disease progression [70-72]. However, improvement in body composition in the absence of weight loss can indicate a healthy change in the ratio of fat to lean muscle, which often accompanies significant changes in diet and physical activity and results in metabolic improvement with enduring benefits [73, 74]. At each assessment point, caregivers and children report on health-promoting family behaviors; mealtime, sleep, and media routines; child and family physical activity habits; child food and beverage choices; and use of community support services. The FITs are used for observational assessment of parenting and family management skills using the validated Coder Impressions Inventory [75] (Dishion T, Hogansen J, Winter C, Jabson J. The coder impressions inventory, unpublished manual). Although not central to our aims, we will also assess several other constructs related to pediatric obesity: weight-related stigma, body image, quality of life, child behavioral and emotional adjustment, and caregiver satisfaction with services.

\section{Data analysis}

\section{Overview}

Analyses focus on implementation data from the families randomized to the FCU4Health. Data from families receiving services as usual will be used in aims $2 \mathrm{~b}$ and 3 . Before data analysis, we will test the psychometric properties (i.e., distribution, reliability) of the measures, conduct a sequence of nested multiple-group confirmatory factor analyses [76] to ensure measurement invariance across language and ethnicity, and apply state-of-the-science data reduction techniques (confirmatory factor analysis, weighted regression scores) by creating multi-indicator, multi-informant construct variables when applicable. These techniques improve measurement properties and result in fewer contrasts, reducing type I error [77]. We expect the attrition rate across waves to be $10-15 \%$ based on retention rates for local studies with highly mobile, hard-to-track samples [78, 79]. We will adjust for missing data due to attrition using multiple imputation [80] and full information maximum likelihood [81]. We will perform sensitivity analyses to check the non-response mechanism [82] and attrition analyses to detect differential attrition rates and attrition by group interaction on sociodemographic and pretest variables that may pose a threat to the validity or the equity of the findings [83]. When missingness is not ignorable, we will use complex models to obtain parameter estimates [84]. To estimate power, we used Optimal Design [85] and Monte Carlo simulations in Mplus [84] and referred to relevant publications. All power analyses used $1-\beta=.80$ and $\alpha=.05$ using twotailed tests. For mediation models, we applied simulation in Mplus for estimating the power of path coefficients and referred to a work by Fritz and MacKinnon [86] for examining significance of mediation effects.

For qualitative data analysis, stakeholder and caregiver interviews and $\mathrm{CAB}$ discussions will be audio recorded, transcribed, and then coded using a directed content analysis approach [87] by using existing theory, prior results, and empirical frameworks to develop a coding scheme. Codes will be refined using a subsample of interviews, and then a team of trained coders will code full transcripts. Twenty percent of interviews will be double coded in order to calculate reliability [88]. Disagreements in coding will be resolved via consensus.

\section{Aim 1}

Results of the adaptation activities are largely descriptive and involve the use of qualitative data from the $C A B$ small group discussions to describe the process and content of adaptations to the program during the course of the trial. 


\section{Aim 2}

In addition to the specific outcomes in aims $2 a-2 c$, we will compare the two arms on stakeholder and caregiver acceptability using repeated-measures analysis of variance (ANOVA) to analyze scores of approximately 30 stakeholders across two time points. We have sufficient power to detect a medium effect for this analysis $(f \geq .32)$. Concerning the variance of caregiver acceptability ratings between groups or over time, we have sufficient power to detect a medium effect $(f \geq .28)$. Latent growth curve (LGC) analyses will be used to determine group differences in the trajectory over time. The estimated power for this analysis is $\beta=0.12$.

\section{Aim 2a: fidelity}

We will calculate the mean score, interrater correlation coefficient, and internal consistency of the observational ratings. Using a mixed-effects model with random effects for families and a fixed-effects model, we will compare scores from prior FCU trials [53-55, 89] (Smith JD, Dishion TJ, Rudo-Stern J, Stormshak EA, Brown K, Ramos K, Thornton N, Shaw DS, Wilson MN, , A quasi-experimental study of the sensitivity and efficiency of observationally assessing fidelity, submitted) to those from the current trial. We have power to detect a small effect of $d \geq .18$. Second, we will use the LGC analysis to (1) evaluate drift in fidelity [53] (power to detect a small effect of $d \geq .16$ for $\mu_{\text {slope }}$ ) with the sample size of 80 families and 240 feedback sessions and (2) assess the relation between variability in fidelity on program effects (power to detect a small effect of $\beta \geq .10$ for $\mu_{\text {slope }}$ on outcomes). To evaluate fidelity using automated coding, we will conduct the same analyses as above but with all 600 feedback sessions. Even when controlling for imperfect reliability, we have power to detect a small effect of $d \geq .10$ for comparison to previous trials, a small effect of $d \geq .11$ for $\mu_{\text {slope }}$, and a small effect of $\beta \geq .08$ for $\mu_{\text {slope }}$ on outcomes.

\section{Aim 2b: costs}

An ingredient-based cost analysis procedure will be employed to estimate FCU4Health installation and continued implementation costs. Using an activity-based costing approach will allow us to value activities both locally and from national data sources (e.g., US Bureau of Labor Statistics), providing estimates relevant for scale-up in new pediatric care settings nationwide. First, we will conduct a budget impact analysis (BIA) of the cost to install the program (i.e., prior to enrollment and provision of care). BIA provides an estimate of the financial consequences of adopting a new intervention from the perspective of the entity financing the intervention [90]. Second, a multi-perspective cost-benefit analysis will be undertaken to capture the potential impact of the
FCU4Health on different key stakeholders (i.e., payor, hospital, family). The fiscal models will be structured to estimate monetary benefits associated with program effects. For significant program effects on the outcome measures in aims 1 and 2, we will calculate the total benefit as a function of the size of the effect $(Q)$ and the price per unit $(P)$ for each year $(y)$. This value can be simply represented as $B_{y}=Q_{y} \times P_{y}$. Effects at 6 and 12 months will be adjusted using a discount rate to accommodate the impact of time on our estimates. To avoid overlap or double-counting benefits, we will employ a weighted average approach of outcomes [91]. We will calculate the average based on the total effect sizes for each outcome in order to estimate the benefits from a single monetary source. While point estimates often represent the bottom line for such an evaluation (e.g., a return-on-investment amount), a confidence interval that incorporates different values of model input that might feasibly occur is more appropriate given the various model assumptions used [92]. A common model input that could take a range of values represents economic uncertainty (i.e., the discounting rate) [93]. In addition to representing the variation that might be expected due to sampling, we will also consider a range of estimates due to anticipated variation in program characteristics. We will consider how key aspects of the delivery system, such as facility costs or provider salary, might vary with new installations.

\section{Aim 2c: sustainability}

The sustainability plan will be largely descriptive and involve the synthesis of quantitative and qualitative data across implementation outcomes, caregiver satisfaction survey results, and $\mathrm{CAB}$ guidance. We will use a mixedmethods data analytic approach (QUANT = QUAL) [94] to develop the sustainability plan.

\section{Aim 3}

We will apply intent-to-treat analyses, using data from all participants who are randomly assigned to the two arms. Our unbalanced design (200 FCU4Health, 150 services-as-usual) only slightly affects statistical power [95]. The primary outcomes are the distal effects on child weight status and body composition and effects on more proximal mediators, including healthy lifestyle (dietary and physical activity habits), child selfregulation, parenting variables, and use of community support services. The LGC analysis for the continuous BMI and body composition measures will be used to compare the trajectories of the two conditions over time analyzed with random-effects models [96]. Specifically, we will estimate whether individual changes (i.e., growth trajectories) over time vary from person to person and whether inter-individual variation is systematically 
related to intervention assignment. For comparing FCU4Health and control arms using analysis of covariance (ANCOVA), we have power to detect a mean difference with a small-to-medium effect size $(d \geq 25$; odds ratio $\geq 1.5$, assuming the base rate of the control group is $30 \%$ ). This means we have power to detect the size of effect on BMI found in previous trials with children and early adolescents, which varies significantly from small to medium (range: $d=.20-.40$ ) across intervention types [97-100] but is large $(d \geq .50)$ for familycentered behavioral programs [101, 102]. For the growth models (repeated-measures analysis using three or four data points), we have power to detect a small effect on the trajectory $\left(\beta=.12\right.$ or $\left.\eta^{2} \leq 2 \%\right)$. LGC modeling has better power than the two-time point ANCOVA for detecting treatment effects in a randomized design [103]. Second, we will examine whether program effects on the distal outcomes are transmitted through the program effects on the proximal variables using mediation analyses, controlling for baseline measures of the mediator and outcome. Bootstrapping will be used to form confidence intervals to test for mediation effects, which has been shown to have better power $[86,104]$. We have power to detect a mediated effect if the two paths each have a small-to-medium effect ( $\beta$ values $\geq .14$ ).

Baseline weight, ecological risk, minority status, poverty status, and child gender will be included as covariates and tested as potential moderators. Moderation of program effects will be examined by adding a program by moderator interaction in the models of primary outcomes and mediators one at a time. For significant interactions, post hoc probing of simple effects (i.e., effects at different values of the moderator) will be conducted, following Aiken and West [105]. Accounting for the imperfect reliability, we have power to detect moderation effects that account for $R^{2} \leq 3 \%$ of the outcome [106].

We will examine whether it is necessary to account for site and coordinator effects by calculating the design effect metric [107]. If significant, we will modify our statistical plans and utilize multi-level models with site or coordinator as the cluster variable.

\section{Aim 4}

To determine differences in family participation between conditions, we will use the Wilcoxon rank-sum tests. We have sufficient power to detect a medium effect for this analysis $(f \geq .30)$. Next, we will use path analysis in structural equation modeling to test a model of program implementation where family engagement mediates the relation between fidelity and clinical outcomes [58]. Using automated fidelity data on the FCU4Health condition sample $(N=200)$, we have power to detect a mediated effect if the two paths each has a small-to-medium effect ( $\beta$ values $\geq .21$ ).

\section{Study status}

At the time this manuscript was submitted for publication, the study was underway. We are planning for our fourth $\mathrm{CAB}$ meeting; initial adaptation of the program and assessment is complete; interviewers, coordinators, and other program staff have been trained; and recruitment is in progress at all three agencies. We have also begun formative work on the development of the automated fidelity coding system using data from previous FCU trials.

\section{Discussion}

Similar to some previously published study protocols in this journal, our type II hybrid effectiveness-implementation trial blends pragmatic trial and implementation science frameworks. However, our protocol is somewhat unique to the hybrid trial design described by Curran et al. [46] because the two implementation strategies differ on the pragmatic-explanatory trial continuum [108] in which the integrated/co-located care arm of the trial is more pragmatic compared to the coordinated care with referral condition, which is more explanatory. However, both conditions are far more pragmatic than they are explanatory-as would be expected for a hybrid trial. This was done to speed translation given that the effectiveness of the adapted FCU4Health program was partially supported by the large existing corpus of evidence supporting the original FCU to both change the purported mediators as well as the primary child outcomes [44]. Additionally, the request for applications for the Childhood Obesity Research Demonstration Projects 2.0 indicated preference for such a design [109].

One of the primary challenges we have encountered since beginning this study relates to working with healthcare organizations. Context is always critical to implementation research, and differences are magnified when attempting to implement a complex behavioral intervention where, previously, a similar program did not exist. The partnerships between the research team and the community sites are critical for success [110], and we have been able to remain successful due to the strength of our partnership, but administrative, personnel, and other issues required extensive meetings, which protracted our planned preparation time from 6 months to nearly 12 months. Many of the issues that were raised could not have been avoided. In part, the extended time was due to variation within our partner clinics that necessitated meetings as well as continually garnering buy-in from leadership and staff that would deliver the FCU4Health.

In conclusion, this study was designed to address a larger issue in the field of pediatric obesity management-the lack of penetration of evidence-based programs into the primary healthcare system. The B rating for family-centered weight management interventions 
for children aged 6 to 12 years with overweight and obesity by the US Preventive Services Task Force [111] provided a financial avenue for such interventions in primary care. This study was aimed at testing and evaluating the many factors that comprise the struggle of making a complex, evidence-based program work in the real world [112]. Achieving wide-scale adoption of the FCU4Health, if found to be effective, would impact the pediatric obesity epidemic in a way that could simultaneously address a number of the factors that result in healthcare disparities for chronic conditions.

\section{Additional file}

Additional file 1: Notice of award. Notice of award from CDC for year 1. (PDF $245 \mathrm{~kb}$ )

\author{
Abbreviations \\ ANCOVA: Analysis of covariance; ANOVA: Analysis of variance; BMI: Body \\ mass index; CAB: Community advisory board; FCU: Family Check-Up; \\ FCU4Health: Family Check-Up 4 Health; FQHC: Federally Qualified Health \\ Center; LGC: Latent growth curve; MI: Motivational interviewing
}

\section{Acknowledgements}

The authors wish to thank our collaborators on this cooperative agreement in the Division at the Centers for Disease Control and Prevention (Brook Belay, Heidi Blanck, Carrie Dooyema, Alyson Goodman, Ann O'Connor); our dedicated study staff at the Arizona State University (Martha Serrano, Lizeth Alonso, Kasha Blair, Monique Lopez, Kirsten Letham, Jon Lipfert, Jenn Yun-Tein), the Northwestern University (Krystal Baker, Katia Chernyshov), and the University of Southern California (Victor Martinez Palacios and colleagues at the Signal Analysis and Interpretation Lab); our partner healthcare agencies; and the many individuals providing guidance and input as members of our community advisory board.

\section{Funding}

This study is supported by grant U18 DP006255 from the National Center for Chronic Disease Prevention and Health Promotion of the Centers of Disease Control and Prevention, under the Childhood Obesity Research Demonstration Project 2.0 (CORD), awarded to Cady Berkel and Justin Smith. Additional support was provided by grant DA027828 from the National Institute on Drug Abuse, awarded to C. Hendricks Brown, and by the Implementation Research Institute (IRI) at the George Warren Brown School of Social Work, Washington University in St. Louis, through grant R25 MH080916 from the National Institute of Mental Health and the Department of Veterans Affairs, Health Services Research and Development Service, Quality Enhancement Research Initiative (QUERI). The opinions expressed herein are the views of the authors and do not necessarily reflect the official policy or position of the Centers for Disease Control and Prevention, the Department of Veterans Affairs, the National Institute on Drug Abuse, the National Institute of Mental Health, or any other part of the US Department of Health and Human Services. The development work for this study was supported by a research grant from the College of Liberal Arts and Sciences at the Arizona State University, awarded to Thomas Dishion.

\section{Availability of data and materials}

Physicians and other staff in the agencies will have access to personal data of their own patients. Anonymized final trial dataset will be accessed only by members of the research team named on the approved IRB protocol. Investigators will offer the possibility of communicating trial results to participants and healthcare professionals under request. Anonymized trial results will be updated in Clinicaltrials.gov for the general public.

\section{Authors' contributions}

JDS and CB conceived of the study. JDS and CB designed and coordinated the study. NJ, CG, DCA, SSN, TJD, AMM, JR-S, KJG, and EW participated in the different phases of the study design. JDS, CB, TJD, AMM, JR-S, and MMB developed the different aspects of the clinical intervention and its related training components and delivery procedures. JDS, CB, TJD, NJ, MKM, DCA and SSN collaborated in the writing of the manuscript. JDS and CB wrote the final manuscript. All authors read and approved the final manuscript.

\section{Ethics approval and consent to participate}

The trial is designed in accordance with the basic ethical principles of autonomy, beneficence, justice, and non-maleficence and will be conducted in accordance with the rules of Good Clinical Practice outlined in the most recent Declaration of Helsinki. Written informed consent of patients will be required. Data confidentiality and anonymity will be ensured, according to the provisions of US law, both during the implementation phase of the study and in any resulting presentations or publications.

The study has been favorably evaluated by the Institutional Review Board of Arizona State University on July 14, 2016 (Protocol 00004530), and by the Institutional Review Board of the Phoenix Children's Hospital on May 30, 2017 (Protocol 17-001). All other institutions participating in this research provided signed reliance agreements ceding to the Institutional Review Board of Arizona State University. A model of the informed consent completed by the participants of this trial is provided as an Additional file 1. Any important protocol modification will be communicated to the Institutional Review Board of Arizona State University and updated in Clinicaltrials.gov.

\section{Consent for publication}

Not applicable.

\section{Competing interests}

JDS and CB are the co-developers of the Family Check-Up 4 Health program. TJD is the developer of the original Family Check-Up program. DCA and SSN are co-founders with equity stakes in a technology company, Lyssn.io, focused on tools to support training, supervision, and quality assurance of psychotherapy and counseling.

\section{Publisher's Note}

Springer Nature remains neutral with regard to jurisdictional claims in published maps and institutional affiliations.

\section{Author details}

${ }^{1}$ Department of Psychiatry and Behavioral Sciences, Northwestern University Feinberg School of Medicine, Chicago, IL, USA. ${ }^{2}$ Department of Preventive Medicine, Northwestern University Feinberg School of Medicine, Chicago, IL, USA. ${ }^{3}$ Department of Pediatrics, Northwestern University Feinberg School of Medicine, Chicago, IL, USA. ${ }^{4}$ REACH Institute, Department of Psychology, Arizona State University, Tempe, AZ, USA. ${ }^{5}$ Department of Psychiatry and Behavioral Sciences, University of Washington School of Medicine, Seattle, WA, USA. ${ }^{6}$ Department of Electrical Engineering and Computer Science, University of Southern California, CA, Los Angeles, USA. ${ }^{7}$ Department of Nutrition, Arizona State University, Tempe, AZ, USA

Received: 13 November 2017 Accepted: 7 December 2017

Published online: 15 January 2018

\section{References}

1. Barness LA, Opitz JM, Gilbert-Barness E. Obesity: genetic, molecular, and environmental aspects. Am J Med Genet A. 2007;143(24):3016-34

2. Eaton DK, Kann L, Kinchen S, Shanklin S, Flint KH, Hawkins J, Harris WA, Lowry R, McManus T, Chyen D, et al. Youth risk behavior surveillance-United States, 2011. MMWR Surveill Summ. 2012;61(4):1-162.

3. Boutelle KN, Hannan P, Fulkerson JA, Crow SJ, Stice E. Obesity as a prospective predictor of depression in adolescent females. Health Psychol. 2010;29(3):293-8.

4. Crosnoe R, Muller C. Body mass index, academic achievement, and school context: examining the educational experiences of adolescents at risk of obesity. J Health Soc Behav. 2004;45(4):393-407. 
5. Echeverría SE, Vélez-Valle E, Janevic T, Prystowsky A. The role of poverty status and obesity on school attendance in the United States. J Adolesc Health. 2014;55(3):402-7.

6. Ogden CL, Carroll MD, Lawman HG, Fryar CD, Kruszon-Moran D, Kit BK, Flegal KM. Trends in obesity prevalence among children and adolescents in the United States, 1988-1994 through 2013-2014. JAMA. 2016;315(21):2292-9.

7. Broussard BA, Johnson A, Himes JH, Story M, Fichtner R, Hauck F, BachmanCarter K, Hayes J, Frohlich K, Gray N. Prevalence of obesity in American Indians and Alaska Natives. Am J Clin Nutr. 1991;53(6):1535S-42S.

8. Messiah SE, Arheart KL, Lopez-Mitnik G, Lipshultz SE, Miller TL. Ethnic group differences in cardiometabolic disease risk factors independent of body mass index among American youth. Obesity. 2013;21(3):424-8.

9. Wang Y, Beydoun MA. The obesity epidemic in the United States-gender, age, socioeconomic, racial/ethnic, and geographic characteristics: a systematic review and meta-regression analysis. Epidemiol Rev. 2007;29(1):6-28.

10. Control CfD. Prevention: establishing a holistic framework to reduce inequities in HIV, viral hepatitis, STDs, and tuberculosis in the United States. Atlanta: CDC; 2010

11. Brewis AA. Stigma and the perpetuation of obesity. Soc Sci Med. 2014; 118:152-8.

12. Schulz A, Northridge ME. Social determinants of health: implications for environmental health promotion. Health Educ Behav. 2004;31(4):455-71.

13. Van Ryzin MJ, Nowicka P. Direct and indirect effects of a family-based intervention in early adolescence on parent-youth relationship quality, late adolescent health, and early adult obesity. J Fam Psychol. 2013; 27(1):106-16.

14. Collins KS, Hughes DL, Doty MM, Ives BL, Edwards JN, Tenney K. Diverse communities, common concerns: assessing health care quality for minority americans. Commonwealth Fund: New York; 2002.

15. Lloyd-Jones DM, Hong Y, Labarthe D, Mozaffarian D, Appel L, Van Horn L, Greenlund K, Daniels S, Nichol G, Tomaselli GF, et al. Defining and setting national goals for cardiovascular health promotion and disease reduction: the American Heart Association's strategic impact goal through 2020 and beyond. Circulation. 2010;121(4):586-613.

16. Sung-Chan P, Sung YW, Zhao X, Brownson RC. Family-based models for childhood-obesity intervention: a systematic review of randomized controlled trials. Obes Rev. 2013;14(4):265-78.

17. Barr-Anderson DJ, Adams-Wynn AW, DiSantis KI, Kumanyika S. Familyfocused physical activity, diet and obesity interventions in African-American girls: a systematic review. Obes Rev. 2013;14(1):29-51.

18. Hingle MD, O'Connor TM, Dave JM, Baranowski T. Parental involvement in interventions to improve child dietary intake: a systematic review. Prev Med. 2010;51(2):103-11.

19. Kitzman-Ulrich $H$, Wilson $D$, George $S$, Lawman $H$, Segal M, Fairchild A. The integration of a family systems approach for understanding youth obesity, physical activity, and dietary programs. Clin Child Fam Psychol Rev. 2010; 13(3):231-53.

20. Krebs NF, Jacobson MS, American Academy of Pediatrics Committee on Nutrition. Prevention of pediatric overweight and obesity. Pediatrics. 2003; 112(2):424-30

21. Altman M, Wilfley DE. Evidence update on the treatment of overweight and obesity in children and adolescents. J Clin Child Adolesc Psychol. 2015;44(4):521-37

22. Leslie LK, Mehus CJ, Hawkins JD, Boat T, McCabe MA, Barkin S, Perrin EC, Metzler CW, Prado G, Tait VF, et al. Primary health care: potential home for family-focused preventive interventions. Am J Prev Med. 2016;

23. Prado G, Pantin $H$, Estrada $Y$. Integrating evidence-based interventions for adolescents into primary care. Am J Prev Med. 2015;48(4):488-90.

24. Kjøbli J, Ogden T. A randomized effectiveness trial of brief parent training in primary care settings. Prev Sci. 2012;13(6):616-26.

25. Bloom B, Jones LI, Freeman G. Summary health statistics for U.S. children: National Health Interview Survey, 2012. Atlanta: Vital health statistics, series 10, number 258: National Center for Health Statistics; 2013.

26. Burwell SM. 2014 annual report on the quality of care for children in Medicaid and CHIP. Washington DC: Department of Health and Human Services; 2014

27. August GP, Caprio S, Fennoy I, Freemark M, Kaufman FR, Lustig RH, Silverstein JH, Speiser PW, Styne DM, Montori VM. Prevention and treatment of pediatric obesity: an endocrine society clinical practice guideline based on expert opinion. J Clin Endocrinol Metab. 2008;93(12):4576-99.
28. Barlow SE. Expert committee recommendations regarding the prevention, assessment, and treatment of child and adolescent overweight and obesity: summary report. Pediatrics. 2007;120(Supplement 4):S164-92.

29. National Research Council and Institute of Medicine. Early childhood obesity prevention policies. Washington, DC: The National Academies; 2011.

30. Kitzmann KM, Beech BM. Family-based interventions for pediatric obesity: methodological and conceptual challenges from family psychology. Couple and Family Psychology. 2011;1(S):45-62.

31. Rhee KE. Childhood overweight and the relationship between parent behaviors, parenting style, and family functioning. Ann Am Acad Pol Soc Sci. 2008;615(1):11-37.

32. Leslie LK, Mehus CJ, Hawkins JD, Boat T, McCabe M, Barkin SL, Perrin EC, Metzler CW, Prado G, Tait VF, et al. Primary health care: potential home for family-focused preventive interventions. Am J Prev Med. 2016;51(4 (supp 2)):S106-18.

33. Turner KM, Shield JP, Salisbury C. Practitioners' views on managing childhood obesity in primary care: a qualitative study. Br J Gen Pract. 2009; 59(568):856-62.

34. Glasgow RE, Emmons KM. How can we increase translation of research into practice? Types of evidence needed. Annu Rev Public Health. 2007; 28:413-33.

35. Chambers DA, Glasgow R, Stange K. The dynamic sustainability framework: addressing the paradox of sustainment amid ongoing change. Implement Sci. 2013:8:117.

36. Boyd-Ball AJ, Dishion TJ, Liddle HA, Rowe C. Family-centered treatment for American Indian adolescent substance abuse: toward a culturally and historically informed strategy. Adolesc Subst Abuse. 2006:423-48.

37. Smith JD, Knoble N, Zerr AA, Dishion TJ, Stormshak EA. Multicultural competence and the Family Check-Up: indirect effect on adolescent antisocial behavior through family conflict. J Clin Child Adolesc Psychol. 2014:43(3):400-14

38. Dishion TJ, Shaw DS, Connell A, Gardner FEM, Weaver C, Wilson M. The Family Check-Up with high-risk indigent families: preventing problem behavior by increasing parents' positive behavior support in early childhood. Child Dev. 2008;79(5):1395-414.

39. Smith JD, Stormshak EA, Kavanagh K. Results of a pragmatic effectivenessimplementation hybrid trial of the Family Check-Up in community mental health agencies. Adm Policy Ment Health Ment Health Serv Res. 2015;42(3):265-78.

40. Dishion TJ, Andrews DW. Preventing escalation in problem behaviors with high-risk young adolescents: immediate and 1-year outcomes. J Consult Clin Psychol. 1995;63(4):538-48.

41. Connell AM, Dishion TJ, Deater-Deckard K. Variable- and person-centered approaches to the analysis of early adolescent substance use: linking peer, family, and intervention effects with developmental trajectories. MerrillPalmer Q. 2006;52:421-48.

42. Stormshak EA, Connell AM, Véronneau M-H, Myers MW, Dishion TJ, Kavanagh K, Caruthers AS. An ecological approach to promoting early adolescent mental health and social adaptation: family-centered intervention in public middle schools. Child Dev. 2011;82(1):209-25.

43. Boyd-Ball AJ. A culturally responsive, family-enhanced intervention model. Alcohol Clin Exp Res. 2003;27(8):1356-60.

44. Smith JD, Montaño Z, Dishion TJ, Shaw DS, Wilson MN. Preventing weight gain and obesity: indirect effects of a family-based intervention in early childhood. Prev Sci. 2015;16(3):408-19.

45. Dishion TJ, Stormshak EA, Kavanagh K. Everyday parenting: a professional's guide to building family management skills. Champaign: Research; 2011.

46. Curran GM, Bauer M, Mittman B, Pyne JM, Stetler C. Effectivenessimplementation hybrid designs: combining elements of clinical effectiveness and implementation research to enhance public health impact. Med Care. 2012;50(3):217-26.

47. Ware JH, Hamel MB. Pragmatic trials — guides to better patient care? N Engl J Med. 2011:364(18):1685-7.

48. Kelleher KJ, Campo JV, Gardner WP. Management of pediatric mental disorders in primary care: where are we now and where are we going? Curr Opin Pediatr. 2006;18(6):649-53.

49. Kolko DJ, Perrin E. The integration of behavioral health interventions in children's health care: services, science, and suggestions. J Clin Child Adolesc Psychol. 2014;43(2):216-28.

50. Montaño Z, Smith JD, Chiapa A, Miloh T, Dishion TJ: Adaptation and implementation of the Family Check-Up for the treatment of pediatric obesity within a primary care setting. The annual meeting of the Society for Prevention Research: 2014; Washington, DC; 2014. 
51. Proctor EK, Landsverk JA, Aarons GA, Chambers D, Glisson C, Mittman B. Implementation research in mental health services: an emerging science with conceptual, methodological, and training challenges. Adm Policy Ment Health Ment Health Serv Res. 2009;36(1):24-34.

52. Proctor EK, Silmere H, Raghavan R, Hovmand P, Aarons GA, Bunger A, Griffey $R$, Hensley M. Outcomes for implementation research: conceptual distinctions, measurement challenges, and research agenda. Adm Policy Ment Health Ment Health Serv Res. 2011;38(2):65-76.

53. Chiapa A, Smith JD, Kim H, Dishion TJ, Shaw DS, Wilson MN. The trajectory of fidelity in a multiyear trial of the Family Check-Up predicts change in child problem behavior. J Consult Clin Psychol. 2015; Available ahead of print

54. Smith JD, Dishion TJ, Shaw DS, Wilson MN. Indirect effects of fidelity to the Family Check-Up on changes in parenting and early childhood problem behaviors. J Consult Clin Psychol. 2013;81(6):962-74.

55. Smith JD, Dishion TJ, Brown K, Ramos K, Knoble NB, Shaw DS, Wilson MN An experimental study of procedures to enhance ratings of fidelity to an evidence-based family intervention. Prev Sci. 2016;(1):62-70.

56. Atkins D, Steyvers M, Imel Z, Smyth P. Scaling up the evaluation of psychotherapy: evaluating motivational interviewing fidelity via statistical text classification. Implement Sci. 2014;9(1):49.

57. Ritzwoller DP, Sukhanova A, Gaglio B, Glasgow RE. Costing behavioral interventions: a practical guide to enhance translation. Ann Behav Med. 2009;37(2):218-27.

58. Berkel C, Mauricio AM, Schoenfelder EN, Sandler IN. Putting the pieces together: an integrated model of program implementation. Prev Sci. 2011; 12(1):23-33.

59. Dishion TJ, Brennan LM, Shaw DS, McEachern AD, Wilson MN, Jo B. Prevention of problem behavior through annual family check-ups in early childhood: intervention effects from home to early elementary school. J Abnorm Child Psychol. 2014;42(3):343-54

60. Whitlock EP, O'Connor EA, Williams SB, et al. Systematic review for the USPSTF effectiveness of weight management interventions in children: A targeted systematic review for the USPSTF. Pediatrics. 2010;125(2):e396-e418.

61. Smith JD, Berkel C, Hails KA, Dishion TJ, Shaw DS, Wilson MN. Predictors of participation in the Family Check-Up program: a randomized trial of yearly services from age 2 to 10 years. Prev Sci. 2016; e-pub ahead of print

62. Smith JD, Berkel C, Mauricio AM, Rudo-Stern J, Serrano M, Bruening MM, Dishion TJ. Family Check-Up 4 Health (FCU4Health) program overview and protocol for the Raising Healthy Children Study. Tempe: REACH Institute, Arizona State University; 2016.

63. Stiles PG, Boothroyd RA, Snyder K, Zong X. Service penetration by persons with severe mental illness: how should it be measured? J Behav Health Serv Res. 2002;29(2):198-207.

64. Imel ZE, Barco JS, Brown HJ, Baucom BR, Baer JS, Kircher JC, Atkins DC. The association of therapist empathy and synchrony in vocally encoded arousal. J Couns Psychol. 2014;61(1):146-53.

65. Xiao B, Imel ZE, Georgiou PG, Atkins DC, Narayanan SS. "Rate my therapist": automated detection of empathy in drug and alcohol counseling via speech and language processing. PLoS One. 2015;10(12):e0143055.

66. Gallo C, Pantin H, Villamar J, Prado G, Tapia M, Ogihara M, Cruden G, Brown $\mathrm{CH}$. Blending qualitative and computational linguistics methods for fidelity assessment: experience with the Familias Unidas preventive intervention. Adm Policy Ment Health Ment Health Serv Res. 2014;42(5):574-85.

67. Wang D, Ogihara M, Gallo CG, Villamar J, Smith JD, Vermeer W, Cruden G, Benbow N, Brown CH. Automatic classification of communication logs into implementation stages via text analysis. Implement Sci. 2016;11(1):119.

68. Atkins DC, Rubin TN, Steyvers M, Doeden MA, Baucom BR, Christensen A. Topic models: a novel method for modeling couple and family text data. J Fam Psychol. 2012:26(5):816-27.

69. Lord SP, Sheng E, Imel ZE, Baer J, Atkins DC. More than reflections: empathy in motivational interviewing includes language style synchrony between therapist and client. Behav Ther. 2015:46(3):296-303.

70. Burgert TS, Taksali SE, Dziura J, Goodman TR, Yeckel CW, Papademetris X, Constable RT, Weiss R, Tamborlane WV, Savoye M. Alanine aminotransferase levels and fatty liver in childhood obesity: associations with insulin resistance, adiponectin, and visceral fat. J Clin Endocrinol Metab. 2006; 91(11):4287-94.

71. Kim G, Caprio S. Diabetes and insulin resistance in pediatric obesity. Pediatr Clin N Am. 2011;58(6):1355-61.

72. Olza J, Aguilera CM, Gil-Campos M, Leis R, Bueno G, Martínez-Jiménez MD, Valle M, Cañete R, Tojo R, Moreno LA. Myeloperoxidase is an early biomarker of inflammation and cardiovascular risk in prepubertal obese children. Diabetes Care. 2012;35(11):2373-6.

73. Burke V, Milligan RAK, Thompson C, Taggart AC, Dunbar DL, Spencer MJ, Medland A, Gracey MP, Vandongen R, Beilin LJ. A controlled trial of health promotion programs in 11-year-olds using physical activity "enrichment" for higher risk children. J Pediatr. 1998;132(5):840-8.

74. Carnethon MR, Gidding SS, Nehgme R, Sidney S, Jacobs DR Jr, Liu K. Cardiorespiratory fitness in young adulthood and the development of cardiovascular disease risk factors. JAMA. 2003;290(23):3092-100.

75. Chiapa A, Parra Morris G, Veronneau MH, Dishion TJ. Translational research on parenting of adolescents: linking theory to valid observation measures for family centered prevention and treatment Transl Behav Med. 2016;6(1):90-104

76. Widaman KF, Reise SP. Exploring the measurement invariance of psychological instruments: applications in the substance use domain. In: Bryant KJ, Windle MT, West SG, editors. The science of prevention: methodological advances from alcohol and substance abuse research. Washington DC: American Psychological Association; 1997. p. 281-324.

77. Peter $H$, Westfall $R$, Rom D, Wolfinger $R$, Hochberg Y. Multiple comparisons and multiple tests: using the SAS system. Cary: SAS Institute; 2000.

78. Dishion TJ, Connell AM. An ecological approach to family intervention to prevent adolescent drug use: linking parent engagement to long-term reductions of tobacco, alcohol and marijuana use. In: Heinrichs N, Hahlweg K, Doepfner M, editors. Strengthening families: evidence-based approaches to support child mental health. Munster: Verlag fur Psychotherapie; 2008. p. 403-33.

79. Gonzales NA, Dumka LE, Millsap RE, Gottschall A, McClain DB, Wong JJ, Germán M, Mauricio AM, Wheeler L, Carpentier FD. Randomized trial of a broad preventive intervention for Mexican American adolescents. J Consult Clin Psychol. 2012;80(1):1-16

80. Schafer JL, Graham JW. Missing data: our view of the state of the art. Psychol Methods. 2002;7:147-77.

81. Arbuckle JL. Full information estimation in the presence of incomplete data. In: Marcoulides GA, Schumacker RE, editors. Advanced structural equation modeling: issues and techniques. Mahwah: Lawrence Erlbaum Associates; 1996.

82. Resseguier N, Giorgi R, Paoletti X. Sensitivity analysis when data are missing not-at-random. Epidemiology. 2011;22(2):282.

83. Jurs SG, Glass GV. The effect of experimental mortality on the internal and external validity of the randomized comparative experiment. J Exp Educ. 1971:40(1):62-6.

84. Muthén LK, Muthén BO. Mplus user's guide (version 7.2). Muthén \& Muthén: Los Angeles; 2014

85. Raudenbush SW, Spybrook J, Congdon R, Liu X, Martinez A. Optimal design plus empirical evidence. 3.0 ed; 2011.

86. Fritz MS, MacKinnon DP. Required sample size to detect the mediated effect. Psychol Sci. 2007;18(3):233-9.

87. Hsieh H-F, Shannon SE. Three approaches to qualitative content analysis. Qual Health Res. 2005;15(9):1277-88.

88. Krippendorff K. Agreement and information in the reliability of coding. Commun Methods Meas. 2011;5(2):93-112.

89. Smith JD, Stormshak EA, Kavanagh K. Results of a pragmatic effectiveness-implementation hybrid trial of the Family Check-Up in community mental health agencies. Adm Policy Ment Health Ment Health Serv Res. 2014;42(3):265-78.

90. Budget impact analysis [https://www.herc.research.va.gov/include/page. asp?id=budget-impact-analysis].

91. Lee S, Aos S, Drake E, Pennucci A, Miller M, Anderson L. Return on investment: evidence-based options to improve statewide outcomes. Olympia: Washington State Institute for Public Policy; 2012.

92. Drummond MF, Sculpher MJ, Claxton K, Stoddart GL, Torrance GW. Methods for the economic evaluation of health care programmes. Oxford: Oxford University Press; 2015

93. Haddix AC, Teutsch SM, Corso PS. Prevention effectiveness: a guide to decision analysis and economic evaluation. Oxford: Oxford University Press; 2003.

94. Palinkas LA, Aarons GA, Horwitz S, Chamberlain P, Hurlburt M, Landsverk J. Mixed method designs in implementation research. Adm Policy Ment Health Ment Health Serv Res. 2011;38(1):44-53.

95. Konstantopoulos S. Power analysis in two-level unbalanced designs. J Exp Educ. 2010;78(3):291-317.

96. Singer JD, Willett JB. Applied longitudinal data analysis: modeling change and event occurrence. New York: Oxford University Press; 2003. 
97. Stice $\mathrm{E}$, Shaw H, Marti CN. A meta-analytic review of obesity prevention programs for children and adolescents: the skinny on interventions that work. Psychol Bull. 2006;132(5):667-91.

98. Kamath CC, Vickers KS, Ehrlich A, McGovern L, Johnson J, Singhal V, Paulo R, Hettinger A, Erwin PJ, Montori VM. Behavioral interventions to prevent childhood obesity: a systematic review and metaanalyses of randomized trials. J Clin Endocrinol Metab. 2008;93(12):4606-15.

99. McGovern L, Johnson JN, Paulo R, Hettinger A, Singhal V, Kamath C, Erwin PJ, Montori VM. Treatment of pediatric obesity: a systematic review and meta-analysis of randomized trials. J Clin Endocrinol Metab. 2008;93(12):4600-5.

100. Cohen J. Statistical power analysis for the bahavioral sciences. 2nd ed. Hillsdale: Erlbaum; 1988.

101. Wilfley DE, Tibbs TL, Van Buren D, Reach KP, Walker MS, Epstein LH. Lifestyle interventions in the treatment of childhood overweight: a meta-analytic review of randomized controlled trials. Health Psychol. 2007;26(5):521-32.

102. Young KM, Northern JJ, Lister KM, Drummond JA, O'Brien WH. A metaanalysis of family-behavioral weight-loss treatments for children. Clin Psychol Rev. 2007;27(2):240-9.

103. McClelland GH, Judd CM. Statistical difficulties of detecting interactions and moderator effects. Psychol Bull. 1993;114(2):376.

104. Taylor AB, MacKinnon DP, Tein J-Y. Tests of the three-path mediated effect. Organ Res Methods. 2007;11(2):241-69.

105. Aiken LS, West SG. Multiple regression: testing and interpreting interactions. Thousand Oaks: Sage; 1991.

106. Aiken LS, West SG. Multiple regression: testing and interpreting interactions. Newbury Park: Sage; 1991.

107. Muthén BO, Satorra A. Complex sample data in structural equation modeling. In: Marsden PV, editor. Sociological methodology. Oxford: Blackwell; 1995. p. 267-316.

108. Loudon K, Treweek S, Sullivan F, Donnan P, Thorpe KE, Zwarenstein M. The PRECIS-2 tool: designing trials that are fit for purpose. BMJ. 2015;350:h2147.

109. Centers for Disease Control and Prevention: Childhood obesity research demonstration 2.0. 2016.

110. Brown CH, Kellam SG, Kaupert S, Muthen BO, Wang W, Muthen LK, Chamberlain P, PoVey CL, Cady R, Valente TW, et al. Partnerships for the design, conduct, and analysis of effectiveness, and implementation research: experiences of the prevention science and methodology group. Admin Pol Ment Health. 2012;39(4):301-16

111. U. S. Preventive Services Task Force, Grossman DC, Bibbins-Domingo K, Curny SJ, Barry MJ, Davidson KW, Doubeni CA, Epling JW Jr, Kemper AR, Krist AH, et al. Screening for obesity in children and adolescents: US Preventive Services Task Force recommendation statement. JAMA. 2017;317(23):2417-26.

112. Brown CH, Curran G, Palinkas LA, Aarons GA, Wells KB, Jones L, Collins LM, Duan N, Mittman BS, Wallace A, et al. An overview of research and evaluation designs for dissemination and implementation. Annu Rev Public Health. 2017;20(38):1-22

113. EPISCenter. Annual Survey of Evidence-based Programs (ASEP). University Park: Pennsylvania State University; 2005.

114. Luke DA, Calhoun A, Robichaux CB, Elliott MB, Moreland-Russell S. The program sustainability assessment tool: a new instrument for public health programs. Prev Chronic Dis. 2014;11:E12.

115. Reimers TM, Wacker DP, Cooper LJ, de Raad AO. Acceptability of behavioral treatments for children: analog and naturalistic evaluations by parents. Sch Psychol Rev. 1992;21(4):628-43.

116. Reimers TM, Wacker DP, Cooper LJ. Evaluation of the acceptability of treatments for children's behavioral difficulties. Child Family Behav Ther. 1991;13(2):53-71.

117. Wacker DP, Berg WK, Harding JW, Barretto A, Rankin B, Ganzer J. Treatment effectiveness, stimulus generalization, and acceptability to parents of functional communication training. Educ Psychol. 2005;25(2-3):233-56.

118. Winter C, Dishion TJ. Parent consultant log. Eugene: Child and Family Center, University of Oregon; 2007.

119. Berkel C, Smith JD, Lopez M. Community resources: engagement and adequacy (CREA) and dosage of engagement in community resources (DECR): measures of community health resource utilization. Tempe: REACH Institute, Arizona State University; 2017.

120. Smith JD, Dishion TJ, Brown K, Ramos K, Knoble NB, Shaw DS, Wilson MN. An experimental study of procedures to enhance ratings of fidelity to an evidence-based family intervention. Prev Sci. 2016;17(1):62-70.
121. CDC/NCHS: CDC growth charts: United States. http://www.cdc.gov/ growthcharts. Posted May 30, 2000. 2000.

122. National Cancer Institute DoCCaPS, Applied Research Program Short Dietary Assessment Instruments: Dietary Screener Questionnaire in the NHANES 2009-2010. [http://appliedresearch.cancer.gov/diet/screeners/] (Accessed June 12, 2016). 2013.

123. Moreno JP, Kelley ML, Landry DN, Paasch V, Terlecki MA, Johnston CA, Foreyt JP. Development and validation of the family health behavior scale. Pediatric Obesity. 2011;6(2Part2)

124. Sadeh A, Mindell JA, Luedtke K, Wiegand B. Sleep and sleep ecology in the first 3 years: a Web-based study. J Sleep Res. 2009;18(1):60-73.

125. Walton K, Simpson JR, Darlington G, Haines J. Parenting stress: a crosssectional analysis of associations with childhood obesity, physical activity, and TV viewing. BMC Pediatr. 2014;14(1):244.

126. Metzler CW, Biglan A, Ary DV, Li F. The stability and validity of early adolescents' reports of parenting constructs. J Fam Psychol. 1998;12(4):600-19.

127. McEachern A, Dishion TJ, Weaver CM, Shaw DS, Wilson MN, Gardner FEM. Parenting Young Children (PARYC): validation of a self-report parenting measure. J Child Fam Stud. 2012;21(3):498-511.

128. Criss MM, Shaw DS. Sibling relationships as contexts for delinquency training in low-income families. J Fam Psychol. 2005;19(4):592-600.

129. Dishion TJ, Mun CJ, Tein J-Y, Kim H, Shaw DS, Gardner F, Wilson MN, Peterson J. The validation of macro and micro observations of parent-child dynamics using the relationship affect coding system in early childhood. Prev Sci. 2017;18(3):268-80.

130. Putnam SP, Rothbart MK. Development of short and very short forms of the children's behavior questionnaire. J Pers Assess. 2006;87(1):102-12.

131. Ellis LK, Rothbart MK: Revision of the early adolescent temperament questionnaire. 2001 biennial meeting of the Society for Research in child development: 2001; Minneapolis, MN; 2001.

132. López-Guimerà G, Fauquet J, Sánchez-Carracedo D, Barrada J, Saldaña C, Masnou-Roig A. Psychometric properties of the perception of teasing scale in a Spanish adolescent sample: POTS-S. Eat Weight Disord. 2012;17(3):e210-8.

133. Truby H, Paxton SJ. Development of the children's body image scale. Br J Clin Psychol. 2002:41(2):185-203.

134. Varni JW, Seid M, Kurtin PS. PedsQL ${ }^{\text {TM }}$ 4.0: reliability and validity of the Pediatric Quality of Life Inventory ${ }^{\mathrm{TM}}$ version 4.0 Generic Core Scales in healthy and patient populations. Med Care. 2001;39(8):800-12.

135. Nguyen TD, Attkisson CC, Stegner BL. Assessment of patient satisfaction: development and refinement of a service evaluation questionnaire. Eval Program Plann. 1983;6(3-4):299-313.

136. Austin CA, Finn SF, Keith TZ, Tharinger DJ, Fernando AD. The Parent Experience of Assessment Scale (PEAS) development and relation to parent satisfaction. Assessment. 2016;

137. Goodman R. The strengths and difficulties questionnaire: a research note. J Child Psychol Psychiatry. 1997:38(5):581-6.

\section{Submit your next manuscript to BioMed Central and we will help you at every step:}

- We accept pre-submission inquiries

- Our selector tool helps you to find the most relevant journal

- We provide round the clock customer support

- Convenient online submission

- Thorough peer review

- Inclusion in PubMed and all major indexing services

- Maximum visibility for your research

Submit your manuscript at www.biomedcentral.com/submit 